\title{
STUDIES ON THE CONCENTRATION AND BACTERIAL EFFECT OF AUREOMYCIN IN DIFFERENT PORTIONS OF THE INTESTINAL TRACT
}

\author{
By LEON V. McVAY, JR. \\ (From the Division of Medicine of the University of Tennessee College of Medicine and the \\ John Gaston Hospital, Memphis, Tennessee)
}

(Submitted for publication July 23, 1951; accepted October 9, 1951)

Numerous investigations have been carried out dealing with the absorption of aureomycin into the blood stream, body fluids, and tissues $(1,2)$. However, no determination of the concentration of this antibiotic within the lumen of the intestine has been reported. Likewise, there have been many studies concerning the effect of oral aureomycin on the intestinal bacterial flora, but these have relied upon the examination of stool specimens (3). It was believed that a determination of the concentration of aureomycin and its bacterial effect in various portions of the intestine would be of value in establishing more accurately the role of this antibiotic in intestinal disease.

For these reasons, it was decided to investigate patients having a cecostomy, a transverse colostomy or a sigmoid colostomy. In this manner, it was possible to correlate oral dosage and intestinal aureomycin levels as well as alterations in the bacterial flora in different portions of the intestinal tract. Similar studies were carried out using stool specimens from a group of hospital patients having no apparent intestinal disease. It was recognized that a comprehensive investigation of intestinal bacteria involving aerobic and anaerobic studies would be an extensive project. A review of the bacteriologic records of the John Gaston Hospital for the five year period from January 1, 1945 to January 1, 1950 disclosed that eight pathogenic organisms were most frequently isolated in cases of peritonitis resulting from perforation of the gastrointestinal tract: Escherichia coli, Streptococcus anaerobius, Bacteroides organisms, Streptococcus pyogenes, Streptococcus viridans, Streptococcus faecalis, Clostridium perfringens, and Aerobacter aerogenes. It was decided to restrict the bacteriologic studies to these eight organisms.

\section{METHODS}

Clinical methods. Nine patients having a cecostomy, a transverse colostomy or a sigmoid colostomy were studied. Two of these patients had a cecostomy; three had a transverse colostomy ; and four had a sigmoid colostomy. The following procedure was carried out in every case: after obtaining two control specimens, each patient was given $250 \mathrm{mg}$. of aureomycin orally at 10:00 a.m., 2:00 p.m., 6:00 p.m., and 10:00 p.m. for two days; then, four days after the intestinal or fecal aureomycin had disappeared, this procedure was repeated using $500 \mathrm{mg}$. instead of $250 \mathrm{mg}$. of aureomycin; again, after the aureomycin level in the intestine or feces had been nil for four days, $750 \mathrm{mg}$. of aureomycin were administered four times a day for two days. Medication was followed in every instance with $100 \mathrm{cc}$. of milk. This particular regimen was adopted because it corresponded to the routine antiamebic treatment employed at the John Gaston Hospital (4). Intestinal specimens were obtained at approximately the same period every 24 hours. One of the two cecostomy patients was unable to remain in the hospital more than 10 days. Therefore, only the $2 \mathrm{gm}$. regimen was carried out in that case.

In addition to the nine cecostomy and colostomy cases, 20 hospital patients having no apparent gastro-intestinal disease were investigated following the oral administration of aureomycin as described above. Efforts were made to obtain stool specimens daily for five days in each of these cases. Four of these patients were subjected to the $1 \mathrm{gm}$. regimen, nine to the $2 \mathrm{gm}$. regimen and seven to the $3 \mathrm{gm}$. regimen. Attention was especially directed toward the $2 \mathrm{gm}$. group because it corresponded to our routine anti-amebic therapy (4).

Assay methods. The method employed for determining aureomycin concentration in feces and intestinal material was suggested by Dornbush (5). Four gm. of intestinal material or feces were placed in a large centrifuge.tube; then, $100 \mathrm{cc}$. of acid acetone were added and, after mixing well, this combination was allowed to stand for 10 minutes. Following this, centrifugation was carried out for 10 minutes at 15,000 r.p.m. Thereafter, 1 cc. of the supernatant was withdrawn and used for serial twofold dilutions in Penassay broth. Twenty tubes were employed for each determination. The first tube contained $0.5 \mathrm{cc}$. of undiluted supernatant, tubes 2 through 19 contained $0.5 \mathrm{cc}$. of serial dilutions of the supernatant, and the last tube contained broth alone as a bacterial control. 
When a high concentration of aureomycin was suspected, a 1:10 dilution of the original extraction material was prepared and $1 \mathrm{cc}$. of this was treated exactly as was the undiluted material. After carrying out the above procedure, $1.5 \mathrm{cc}$. of a $1: 100$ dilution of an overnight Penassay broth (Difco) culture of Bacillus cereus was added to each tube and the contents were mixed. Following this, the tubes were incubated at $37^{\circ} \mathrm{C}$. for four hours and 10 minutes. The additional 10 minutes were allowed for heating the tubes initially to $37^{\circ} \mathrm{C}$. This entire process was carried out in duplicate.

After incubation, the tubes were read for the amount of aureomycin as is described below. In every instance, two control series were carried out. The first of these utilized crystalline aureomycin in 2 mcg. per cc. concentration. This portion of the determination was conducted exactly as above except that $1 \mathrm{cc}$. of Penassay broth (Difco) containing 2 mcg. of aureomycin was employed instead of the extraction material. The aureomycin control usually revealed absence of growth through tube 8 , so that inhibition through tube 7 in the assay determination represented $1 \mathrm{mcg}$. of aureomycin per cc. of the extraction material (or $1 \mathrm{mcg}$. of aureomycin per $0.04 \mathrm{gm}$. of intestinal or fecal material). In rare instances, there was a variation of one tube in the aureomycin control determination. The tests were read as follows (for this illustration, it is assumed that inhibition in the aureomycin control series extended through tube 8):

Tube: 1234567891011121314151617181920 Growth: + 1 mcg. aureomycin per $0.04 \mathrm{gm}$. of intestinal or fecal material; that is, 1 mcg. per cc. of extraction material)

$++(0.5$ mcg. of aureomycin per 0.04 gm. of fecal or intestinal material)

$+++(0.25 \mathrm{mcg}$. of aureomycin per 0.04 gm. of fecal or intestinal material).

The second control was performed exactly as the first except that $1 \mathrm{cc}$. of acid acetone was employed, instead of 2 mcg. of crystalline aureomycin in $1 \mathrm{cc}$. of Penassay broth. Inhibition in this control series varied from tubes 1 to 4 . Therefore, whenever in the assay of intestinal or fecal material, inhibition was the same in the test series and in the acid acetone control, the aureomycin concentration was read as 0 . In early determinations, it was found that no difference in levels occurred when the crystalline aureomycin was dissolved in Penassay broth or acid acetone. The acid acetone employed in extracting aureomycin from fecal or intestinal material was prepared as follows: 1 part of $4 \mathrm{~N}$ hydrochloric acid, 6 parts of water and 13 parts of acetone.

In most instances, aureomycin assay determinations were carried out within one hour of the receipt of the intestinal or fecal specimens; in no case was there an interval exceeding two hours. Prior to this procedure the material was maintained at $-20^{\circ} \mathrm{C}$. Two intestinal or fecal specimens were obtained in each case as controls before the initial aureomycin regimen was instituted. No evidence of aureomycin was noted in any of the pre-treatment control specimens. While no history of the recent use of antibiotics or chemotherapeutic agents could be obtained in any patient selected for this investigation, these control specimens provided additional protection against such a possibility.

Bacteriologic methods. Stool and intestinal specimens were collected at approximately the same period every 24 hours in autoclaved dry containers. Cultures were made within one to two hours after the collection of specimens. The following procedure was employed for this purpose: approximately $1 \mathrm{gm}$. of feces or intestinal material was transferred aseptically to $10 \mathrm{cc}$. of sterile saline and thoroughly mixed. One cc. of this material was transferred to a tube containing $9 \mathrm{cc}$. of sterile physiological saline and the contents again were thoroughly mixed. This procedure was carried out through five tubes with the tubes being numbered 1 through 5 consecutively. Fourteen blood agar plates were used for each specimen. These were divided into two sets of seven plates. In each set, the plates were numbered 1 through 7. Two drops of the contents of tube 2 were transferred aseptically to the first blood agar plate and one drop was similarly placed on the second plate. The material was spread evenly on the first plate with a sterile glass spreader. A second spreader was utilized to distribute the material on plate 2 and dilution was then obtained by using the same spreader for the third plate. This procedure was carried out in duplicate. The same method was employed for inoculating three eosin methylene blue plates (Difco). In a similar manner, two drops of the contents of tube 5 were placed on the fourth blood agar plate and the material was then diluted by using the same spreader for the fifth plate. This procedure was also carried out in duplicate. Tube 1 was heated in a water bath at $80^{\circ} \mathrm{C}$. for 15 minutes, after which two drops of its contents were placed on the sixth blood agar plate and were subsequently diluted on the seventh plate by using the same glass spreader. One set of blood agar plates and the three eosin methylene blue plates were incubated aerobically at $37^{\circ}$. C. for 24 hours. The remaining set of blood agar plates was incubated anaerobically at $37^{\circ} \mathrm{C}$. for 48 hours.

The gross appearance of all bacterial colonies was recorded. The first plate was used to determine the number of each type of colony except when it was overgrown. When the first plate could not be employed, the fourth plate was utilized for this purpose; and the number of colonies counted was multiplied by the dilution factor. The amount of growth was recorded as follows:

$$
\begin{aligned}
& \text { Negative-no growth } \\
& 1+\text {-from } 1 \text { to } 15 \text { colonies } \\
& 2+\text {-from } 16 \text { to } 50 \text { colonies } \\
& 3+\text {-from } 51 \text { to } 100 \text { colonies } \\
& 4+\text { - more than } 100 \text { colonies. }
\end{aligned}
$$

\section{RESULTS}

Assay results. Using $1 \mathrm{gm}$. of oral aureomycin daily for two days, intestinal and fecal concentrations of at least $1,000 \mathrm{mcg}$. per gm. of material were obtained for varying periods in every case except one. In three instances maximum aureomy- 
cin concentrations ranged from 51,200 to 102,400 mcg. per gm. Fecal aureomycin levels in four patients, having no cecostomy or colostomy, receiving $1 \mathrm{gm}$. daily for two days, varied from 3,200 to $51,200 \mathrm{mcg}$. per $\mathrm{gm}$. of material.

With $2 \mathrm{gm}$. of oral aureomycin daily, higher concentrations were obtained in the intestine and stool than in the preceding series. Maximal concentrations ranging from 25,600 to $102,400 \mathrm{mcg}$. per gm. of intestinal material were obtained in seven of the nine cecostomy and colostomy patients. Stool aureomycin levels reaching 25,000 to $50,000 \mathrm{mcg}$. per gm. of wet material were obtained in every instance (Table I).

With $3 \mathrm{gm}$. of oral aureomycin daily for two days, persistently higher concentrations were obtained in the intestine and feces than in either of the two preceding groups. Of importance is the fact that significant aureomycin intestinal levels were rapidly attained and were then maintained for two and three days after medication was discontinued. With the exception of the cecostomy case, every patient in this series disclosed intestinal or fecal concentrations of aureomycin reaching 51,600 to $128,000 \mathrm{mcg}$. per gm. of wet material. Since only graded quantitative differences were noted in the results obtained with $1 \mathrm{gm}$., $2 \mathrm{gm}$. and $3 \mathrm{gm}$. of aureomycin, the concentrations demonstrated in the $2 \mathrm{gm}$. series alone are recorded in Table I.

The effect of diarrhea on aureomycin intestinal levels is of obvious practical importance in the treatment of intestinal disease, for example, human amebic dysentery. The rapid decrease in the aureomycin level in patient 3 in the $2 \mathrm{gm}$. series (Table I) was associated with a painless diarrhea of five to seven liquid stools per day. Additional evidence of the effect of diarrhea in reducing aureomycin intestinal levels was observed in the two cecostomy patients in this series (Table I). Patient 1 had a persistent, moderately severe diarrhea throughout this investigation; while the second patient noted no side effects. Concentrations obtained in the latter case were four to 12.5 times greater than in the first patient.

Bacteriologic results. To promote cohesion in discussion and representation, the bacteriologic studies involving the various portions of the intestine-cecum, transverse colon and sigmoid colon -were grouped together as intestinal effects. When a reduction of one of the eight bacteria studied was observed from $4+$ or $3+$ to $1+$ or 0 (as previously explained), it was regarded as evidence

TABLE I

Aureomycin intestinal and stool levels

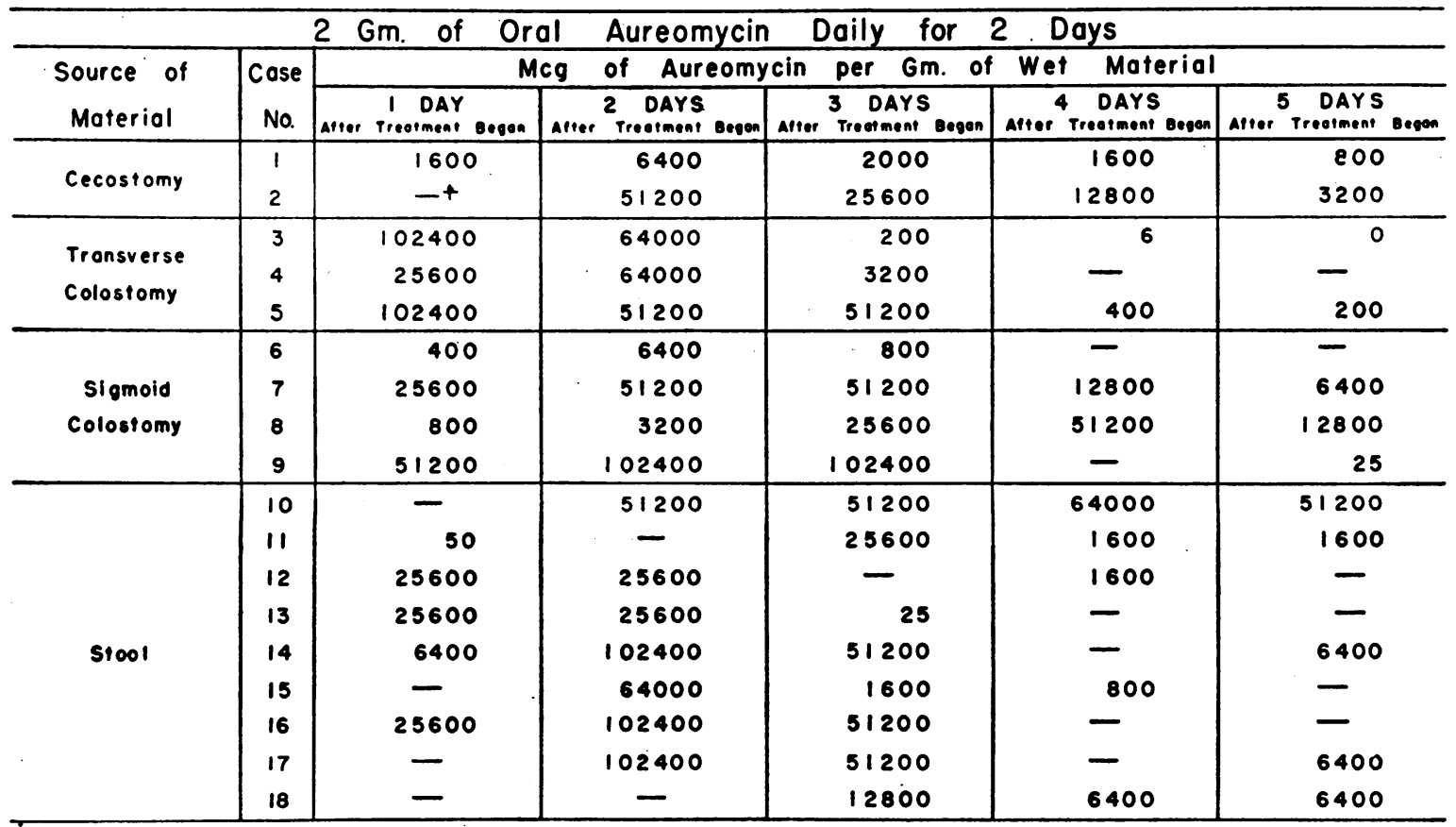

+ Indicates " $C$ " specimens were not obtained. 
TABLE II

Bacteriologic effects

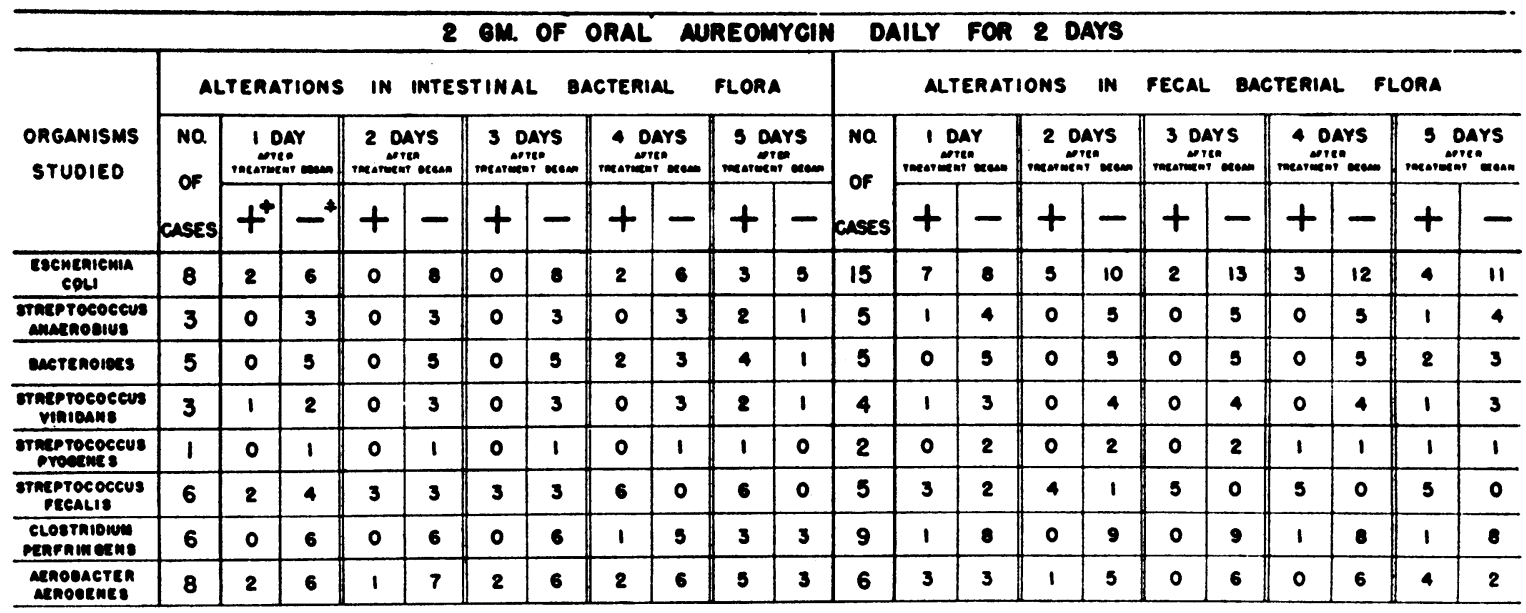

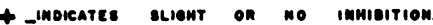

+ imoicates manked on complete immioirion.

of marked or complete inhibition. If reduction was only in the range of $4+$ to $3+$ or $3+$ to $2+$ or $2+$ to $1+$ or $1+$ to 0 or if there was no decrease, it was regarded as slight or no inhibition. This method was employed with both intestinal and fecal specimens.

Even such a minimal amount of oral aureomycin as $1 \mathrm{gm}$. daily for two days, produced a rapid intestinal effect. Seven of the eight bacteria studied were markedly or completely inhibited in 24 to 48 hours and definite reduction of these organisms persisted for two days after this small amount of medication was discontinued. Only Streptococcus faecalis was not uniformly responsive.

Using 2 and $3 \mathrm{gm}$. of aureomycin daily for two days, a more rapid and marked reduction of bacteria occurred. While $1 \mathrm{gm}$. resulted in the complete inhibition of two of the eight bacteria in the intestine in 24 hours, $2 \mathrm{gm}$. completely inhibited three of these organisms (Table II), and $3 \mathrm{gm}$. caused the complete inhibition of six. Similar effects were noted in the fecal specimens. Only Streptococcus faecalis was not uniformly reduced to a significant degree. However, when $3 \mathrm{gm}$. were administered daily for two days, three of five intestinal cases revealed complete or marked inhibition of this organism, though only one of five of the stool specimens disclosed this degree of reduction. In all cases, the effect on Streptococcus faecalis was brief. A definite inhibition of the other seven bacteria in the intestine and stool was evident for 48 and 72 hours after aureomycin was discontinued.

\section{DISCUSSION}

In an effort to evaluate more exactly the role of aureomycin in intestinal disease, material from various portions of the intestine was examined following the administration of therapeutic amounts of this antibiotic. Each specimen was studied to determine the concentration of aureomycin and its effect on eight pathogenic organisms. Using 2 to $3 \mathrm{gm}$. of oral aureomycin daily, intestinal concentrations of this agent were obtained which reached 51,200 to $128,000 \mathrm{mcg}$. per $\mathrm{gm}$. of wet material.

These initial aureomycin intestinal levels are quite suggestive as regards the mode of action of this antibiotic in human amebiasis. In 1949, it was demonstrated that aureomycin is amebicidal in vitro (6). Further evidence indicating a direct effect by this antibiotic on $E$. histolytica in the test tube was presented by Hewitt and his associates who described the complete destruction of amebae by aureomycin in concentrations of 1,000 mcg. per cc. with only 10 minutes of contact (7). More recently, Hall reported that aureomycin destroyed $E$. histolytica trophozoites in vitro in concentrations of $50 \mathrm{mcg}$. per cc. (8).

After in vitro studies, more than 100 cases of 
amebiasis were successfully treated at the John Gaston Hospital with oral aureomycin. However, the exact mode of action of this antibiotic in the body was not clear. There remained the possibility that aureomycin exerted its effect in the body primarily through an indirect action on amebae by altering the intestinal bacterial flora. That this antibiotic has a broad antibacterial spectrum of activity is well established.

The results of the present studies indicate that the oral administration of therapeutic amounts of aureomycin produces intestinal concentrations which are 50 to 100 times greater than established amebicidal levels in vitro $(51,200$ to $128,000 \mathrm{mcg}$. per gm. of wet material). These first aureomycin intestinal levels suggest, therefore, that this agent exerts its major therapeutic effect in human amebiasis through a direct action on $E$. histolytica. It is generally recognized that the eradication of amebic infections requires the destruction of amebae in tissue as well as in the intestinal lumen. In view of the high concentration of aureomycin obtained within the lumen of the colon, it appears probable that amebicidal amounts penetrate the intestinal wall via the vascular system. It is apparent from the present investigation that while diarrhea causes a reduction in the intestinal concentration of aureomycin, levels are still present which are amebicidal in vitro. The alteration of the intestinal bacterial flora may play a part in the destruction of $E$. histolytica in the body, but this would appear to be of secondary importance.

Bacteriologic studies restricted to eight common pathogenic organisms revealed that 2 to $3 \mathrm{gm}$. of oral aureomycin daily for two days resulted in a rapid and marked inhibition of seven of these bacteria. This effect persisted for 48 to 72 hours after medication was discontinued. Streptococcus faecalis alone was not uniformly responsive; though when $3 \mathrm{gm}$. were administered daily, this organism was markedly reduced in more than half of the cases revealing its presence. While the intestinal antibacterial spectrum of aureomycin has been extensively investigated (3), it is believed that information derived from this study of material from different portions of the intestine may be of value in determining more exactly its role in intestinal surgery. In view of the rapid attainment and subsequent maintenance of significant intestinal levels together with its bacterial effects, it appears that aureomycin should be an excellent agent for the preoperative preparation of the intestine. Clinical reports substantiate this view $(9,10)$.

\section{SUMMARY}

1. The oral administration of therapeutic amounts of aureomycin results in the rapid attainment and rather prolonged maintenance of significant intestinal concentrations of this antibiotic.

2. These first aureomycin intestinal levels indicate that this antibiotic probably exerts its major therapeutic effect in human amebiasis by a direct action on $E$. histolytica; though there may be a minimal indirect action on amebae resulting from alteration of the intestinal bacterial flora.

3. The studies reported in this paper dealing with the concentration of aureomycin and its bacteriologic action in various portions of the intestine indicate that this antibiotic should be an effective agent for preoperative preparation of the intestine.

\section{REFERENCES}

1. Doi nbush, A. C., and Pelcak, E. J., The determination of aureomycin in serum and other body fluids. Ann. New York Acad. Sc., 1948, 51, 218.

2. Herrell, W. E., and Heilman, F. R., Aureomycin studies on absorption, diffusion and excretion. Proc. Staff Meet., Mayo Clinic, 1949, 24, 157.

3. Dearing, W. H., and Heilman, F. R., The effect of aureomycin on the bacterial flora of the intestinal tract of man: a contribution to preoperative preparation. Proc. Staff Meet., Mayo Clinic, 1950, 25, 87.

4. McVay, L. V., Jr., Laird, R. L., and Sprunt, D. H., The treatment of amebiasis with aureomycin. South. M. J., 1950, 43, 308.

5. Dornbush, A. C., Personal communication.

6. McVay, L. V., Jr., Laird, R. L., and Sprunt, D. H., A preliminary report of the successful treatment of amebiasis with aureomycin. Science, 1949, 109, 590.

7. Hewitt, R., Wallace, W., and White, E., The effect of aureomycin on Endamoeba histolytica in vitro. Science, 1950, 112, 144.

8. Hall, W. H., The treatment of chronic human amebiasis with aureomycin. New England J. Med., 1951, 244, 495.

9. Yeager, G. H., Byerly, W. L., Jr., and Holbrook, W. A., Jr., Streptomycin, aureomycin and chloromycetin: experimental and clinical comparison. Ann. Surg., 1949, 130, 576.

10. Wright, L. T., Schreiber, H., Metzger, W. I., and Parker, J. W., An evaluation of aureomycin therapy in peritonitis. Surg., Gynec. \& Obst., 1951, 92, 661. 